\title{
An assessment of cable radiation effects on mobile communications antenna measurements
}

\author{
Seppo Saario, David V. Thiel*, Jun W. Lu and Steven G. O'Keefe
}

Radio Science Laboratory, School of Microelectronic Engineering, Griffith University, Nathan Campus, Brisbane, QLD 4111, Australia

\begin{abstract}
Standard methods of antenna assessment require their connection via coaxial cable to a network analyser. For portable communications equipment, this situation is quite different from the standard operation because current in the outer conductor of coaxial cable feeds can significantly distort the impedance, gain and directional characteristics. In this paper, the use of ferrite beads to minimise cable radiation effects is investigated for various configurations using FDTD modelling.
\end{abstract}

\section{Introduction}

The structure of most mobile communications antennas used in portable handsets involves the use of the chassis of the telephone as an earth reference. The chassis conductor is neither an infinite ground plane (as required for an efficient monopole radiator) or a quarter wave monopole identical to the designated monopole antenna. The telephone with antenna is an asymmetric dipole, and the physical characteristics of the chassis (i.e. its size, shape and orientation) play a significant role in determining radiation properties [1]. In a telephone, the handset contains the transmitter and receiver circuit which are not earth referenced as might be the case for many other antenna structures. This presents significant measurement difficulties for a number of reasons. For network analyzer measurements on the antenna structure (e.g. gain, bandwidth and polar pattern), connections are usually via coaxial cables. It is possible for currents to be induced to flow on the outer surface of the shield of the coaxial cable which changes the over-all configuration of the radiating structure. These currents radiate and so influence the measurements. A common method of minimizing cable radiation is to employ ferrite beads on the coaxial cable as close as possible to the feed point of the antenna.

In this paper, the finite difference time domain method of numerical modeling (FDTD) has been employed to investigate a number of these effects including the position of the coaxial feed cable and the position of a ferrite bead on the cable. 


\section{FDTD modelling}

For the numerical simulations the FDTD method was used with Berenger's Perfectly Matched Layer (PML) boundary conditions. The PML boundary condition used had 4 layers, with a parabolic conductivity profile. A frequencydomain near-to-far transformation was used to obtain the far field radiation pattern. The radiation pattern was calculated at $900 \mathrm{MHz}$ and $1.5 \mathrm{GHz}$. Validation of the code, with PML boundary conditions, Sub-Cellular extensions and the far field radiation pattern was addressed in an earlier paper by Saario et. al. [2].

The first model consisted of a quarter wave monopole at $900 \mathrm{MHz}$ directly on the end of a coaxial cable. The quarter wave monopole was constructed in FDTD space using a grid size of $40 \times 40 \times 160$ with space increments of $\Delta x=\Delta y=1.79 \mathrm{~mm}$ and $\Delta z=2.0825 \mathrm{~mm}$. The second model consisted of a quarter wave monopole on a conductive box. It was constructed using a FDTD region of $100 \times 90 \times 150$ Yee cells. The space increments were $\Delta x=$ $\Delta y=\Delta z=2.5 \mathrm{~mm}$. An IBM SP $/ 2$ supercomputer was used for the numerical simulation, time stepping was carried out for 3000 time steps for both cases. The time step was determined by the Courant stability condition.

\section{Antenna investigations}

In the first investigation a simple quarter wave monopole element was located at the end of a coaxial feed with a ferrite bead positioned on the feed cable. The magnetic permeability of the ferrite was taken as $50 \mu_{0}$ and the dielectric constant was $50 \varepsilon_{0}$. The bead provides a discontinuity in the propagation coefficient for current on the outer part of the shield, which results in significant reflection. The electric field polar pattern of the antenna in the horizontal plane (i.e. 90 degrees to the monopole longitudinal axis) is given in Fig. 1 for a variety of bead positions.

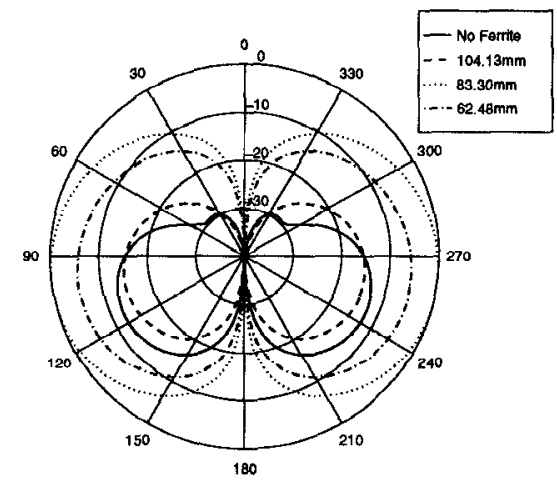

Figure 1: Variation in radiated power with ferrite bead location for a coaxial feed monopole antenna. The length of coaxial cable above the bead is shown. 
In the absence of the ferrite bead, the radiation from the cable is directed 27 degrees below the horizontal plane. When the ferrite bead is positioned on the coaxial cable, the currents in the outer shield of the coaxial cable are confined to that part above the top of the ferrite bead. This results in a dipole in which one portion is the monopole element, and the second is a length of the coaxial cable outer conductor. This interpretation is verified by the change in the polar patterns Fig. 1. Note that maximum radiation efficiency occurs when the bead is $\lambda / 4$ from the end of the outer conductor; i.e. the dipole antenna is almost symmetric. Clearly quite minor changes in the position of the ferrite bead can dramatically affect the radiation efficiency of the antenna.

An alternative view of the behaviour of the antenna structure discussed above, is the similarity between this structure and a sleeve antenna [3] where an additional outer cylinder is coaxially located around the coaxial cable and shorted to it. The ferrite bead appears to be a much simpler method of construction for this type of antenna.

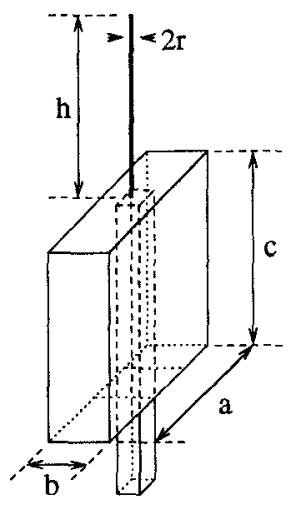

(a)

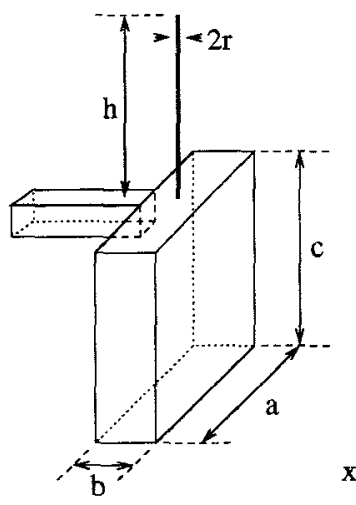

(b) $\mathrm{r}=0.5 \mathrm{~mm}$

$\mathrm{a}=60 \mathrm{~mm}$

$\mathrm{b}=10 \mathrm{~mm}$

$\mathrm{c}=130 \mathrm{~mm}$

$\mathrm{h}=50 \mathrm{~mm}$

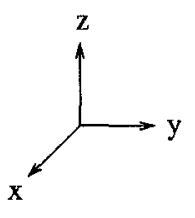

Figure 2: $\mathrm{Z}$ and $\mathrm{Y}$ feed arrangements used in telephone measurements

The second investigation involved two alternative coaxial feed arrangements for a cellular telephone model (conductive box with a quarter wave monopole antenna). Fig. 2(a) shows the $\mathrm{Z}$ feed arrangement and Fig. 2(b) the $Y$ feed arrangement. A ferrite bead was included at a number of positions on the coaxial cable. Results are all compared with the radiation from the telephone model without a feed cable. Fig. 3 indicates that the $\mathrm{Z}$ feed has a significant effect on the polar pattern; particularly in the horizontal plane where the difference is more than $5 \mathrm{~dB}$. The cable also results in a shift in resonant frequency of up to $6 \%$ for both configurations. The $\mathrm{Y}$ feed arrangement is clearly much closer to the isolated case and the presence of the ferrite is almost undetectable in the polar pattern.

Additional investigations involved the size of the ferrite bead and the magnetic permeability of the ferrite. 

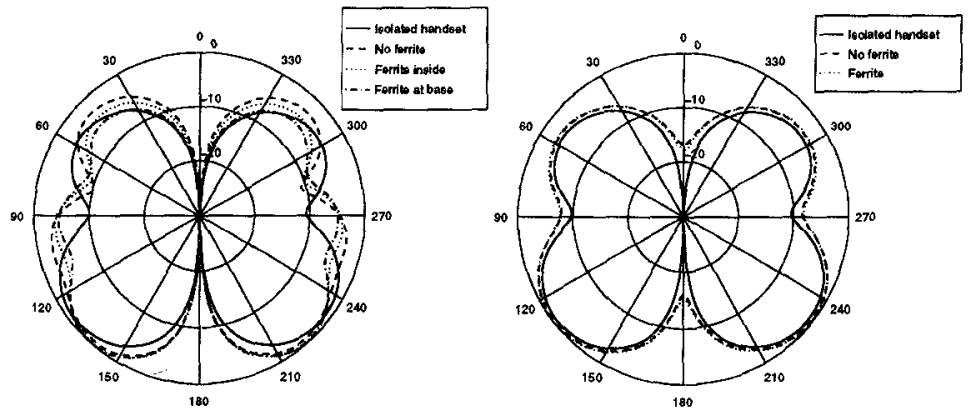

Figure 3: (a) $\mathrm{Z}$ feed and (b) $\mathrm{Y}$ feed radiation characterictics compared to the isolated telephone (solid line)

\section{Conclusion}

Lossless ferrite beads on the outer conductor of a coaxial cable reflect the currents which flow on the outer conductor. The position of the ferrite bead plays and important part in the radiation characteristics of the antenna.

In the case of a telephone handset, the chassis of the telephone usually forms the lower element of an asymmetric dipole. The tuning of the telephone antenna depends on the size and shape of the chassis conductor $[1,4]$. For this reason, the assessment of antennas for hand held portable communications must include the effect of the telephone chassis. If one is to make measurements using a coaxial feed network analyzer, it is particularly important to use the $Y$ feed arrangement.

\section{References}

[1] L. Chen, T. Uno, S. Adachi, R. Luebbers and K. Kunz, "FDTD Method Analysis of a Monopole Antenna Mounted on a Conducting Rectangular Box", IEEE AP-S Int. Symp. Dig., 1670, 1992.

[2] S. Saario, Z. Ma, and E. Yamashita, "FDTD Modelling of Wire Handset Antenna with PML Boundary Conditions and Subcellular Extensions", IEICE Autumn Conference, B-81, Kanazawa, Japan, Sept. 1996.

[3] L. W. Pispin and D C. Chang, "Wire and loop antennas" in "Antenna Handbook" vol.2, ed Y.T. Lo and S.W. Lee, Van Nostrand Reingold, pp. 7-25, New York, 1993.

[4] M. A. Jensen and Y. Rahmat-Samii, "Performance Analysis of Antennas for HandHeld Transceivers Using FDTD”, IEEE Trans. Antennas Propagat., vol. 42, pp 1106-1112, Aug. 1994. 